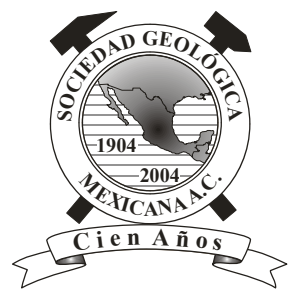

\title{
Editorial \\ El Boletín de la Sociedad Geológica Mexicana ante sus 110 años y su ingreso en el Science Citation Index-Expanded
}

El Boletín de la Sociedad Geológica Mexicana, junto con la Sociedad que le da nombre, cumple, en diciembre del año en curso, 110 años de historia, y lo celebra iniciándolo con la confirmación de su ingreso en el Science Citation IndexExpanded (incluida la Web of Science) y en Current Contents (Physical, Chemical and Earth Sciences). La pertenencia del Boletín en el Science Citation Index-Expanded (SCI) será efectiva a partir del número en que aparece la presente nota editorial: el volumen 66, número 1 de 2014, que constituye un número temático especial sobre Paleobotánica. Dicho ingreso se añade a la integración del Boletín desde años anteriores a otros índices, bases de datos e instrumentos bibliográficos tanto de carácter nacional como internacional: el Índice de Revistas Mexicanas de Investigación Científica y Técnica del CONACYT, Scopus, Scientific Electronic Library Online (SciELO), EBSCOhost, Red de Revistas Científicas de América Latina y el Caribe, España y Portugal (REDALYC), Latindex, Ulrich's, SCImago, Periódica, Directory of Open Access Journals (DOAJ), Dialnet, Zoological Record, UPCommons, Open J-Gate, y Geoscience e-Journals. Tal grado de internacionalización del Boletín y sus contenidos constituye un hito en la historia de la revista y el fruto de un largo esfuerzo que se inició hace 10 años con la edición conmemorativa del centenario de la Sociedad Geológica Mexicana, publicada entre 2005 y 2006. En estos 10 años el Boletín pasó de ser una revista con un futuro incierto - a tenor del escaso interés que suscitaba entre los autores potenciales-a ubicarse nuevamente como una revista de referencia en las Ciencias de la Tierra en México y, además, con una proyección y una visibilidad internacional sin precedentes en su larga historia. Más allá del ingreso en el SCI del Boletín, es preciso resaltar el ejercicio sostenido del comité editorial y de su equipo de edición técnica, así como la estabilidad y efectividad de su línea editorial. Tales elementos han constituido los auténticos artífices de la recuperación del Boletín como revista científica arbitrada, aun cuando se partía de condiciones muy difíciles.

La internacionalización del Boletín puede apreciarse igualmente en la composición de su comité editorial actual,

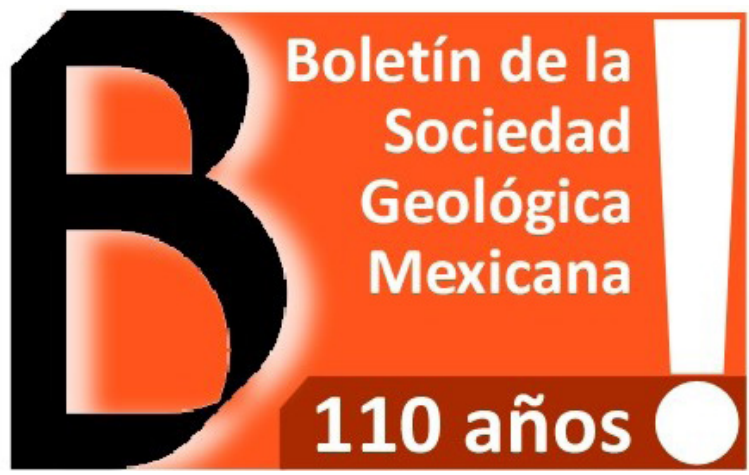

la extracción de los autores publicados (Figura 1), y su padrón de revisores (Figura 2). Nótese que, desde 2006, los arbitrajes de artículos por parte entidades de otros países constituyen un $50 \%$, y que se mantiene un cierto balance entre revisores pertenecientes a la entidad de edición del Boletín (Instituto de Geología de la UNAM; $12 \%$ ), al resto de dependencias de la UNAM (17\%), y a otras entidades mexicanas (21\%). A partir de la edición conmemorativa del centenario de la Sociedad, se han publicado 231 artículos repartidos en 8 volúmenes y 20 números (durante menos de 8 años), con casi 900 autores de alrededor de 200 entidades de adscripción distintas distribuidas en 26 países, en que los autores de fuera de México representan casi el $30 \%$ de los autores publicados (Figura 1). Ese porcentaje se eleva a casi un $32 \%$ teniendo en cuenta el total de autores de manuscritos sometidos (de los que se ha rechazado casi un tercio desde 2006). La cantidad de artículos publicados desde 2006 representa el $33.8 \%$ del total histórico de contribuciones publicadas en el Boletín desde 1904, a pesar de que nunca, hasta el momento presente, había sido una revista tan plural e internacional.

Sin embargo, estos datos no son más que indicadores rápidos del estado actual de la gestión editorial de esta revista científica, pues ello está basado en la mejora sustancial en todos los aspectos de su edición. Entre ellos, son destacables los siguientes: 
1. El Boletín es ahora una publicación electrónica de acceso libre e irrestricto sin mediar pago alguno de cuotas ni para los autores ni para los lectores.

2. La publicación se ha regularizado e instrumentalizado mediante la edición, en gran manera, de números especiales temáticos, con la publicación continua de otros artículos, eventualmente conformando también números regulares.

3. Se ha maximizado la eficiencia y rigor en el proceso editorial manteniendo un equilibrio entre revisores expertos y la pluralidad de sus entidades de adscripción.

4. El Boletín presenta estándares de calidad científica y formal validados por los principales índices e instrumentos bibliográficos nacionales e internacionales.

5. Se ha maximizado la visibilidad del Boletín y sus contenidos, aunque este aspecto todavía posee mucho margen de mejora.

6. Se han extendido las autorías y el padrón de revisores a la gran mayoría de instituciones mexicanas en que se desarrolla investigación dentro del ámbito de las Ciencias de la Tierra.

7. Se ha producido una internacionalización creciente de las autorías y del padrón de revisores.

8. Contribución a la formación de recursos humanos incluso dentro del mismo equipo de edición técnica.

9. Se registra un aumento sustancial en la captación espontánea de artículos, motivada por el aumento de la visibilidad nacional e internacional del Boletín y por su pertenencia en ciertos índices bibliográficos.

10. Se registra un aumento en la variabilidad temática de los artículos, incluyendo áreas de conocimiento de las Ciencias de la Tierra en que históricamente el Boletín había incursionado poco o nada (e.g., Geoarqueología, Paleoclimatología, Geología Urbana, Paleontología, Geología Marina, Edafología, etc.)

11. Mantenimiento eficiente de la periodicidad cuatrimestral (abril, agosto y diciembre) con puntualidad perfecta.

12. Se registra un aumento sustancial general en la cantidad de contribuciones al Boletín y en sus contenidos publicados y, en consecuencia,

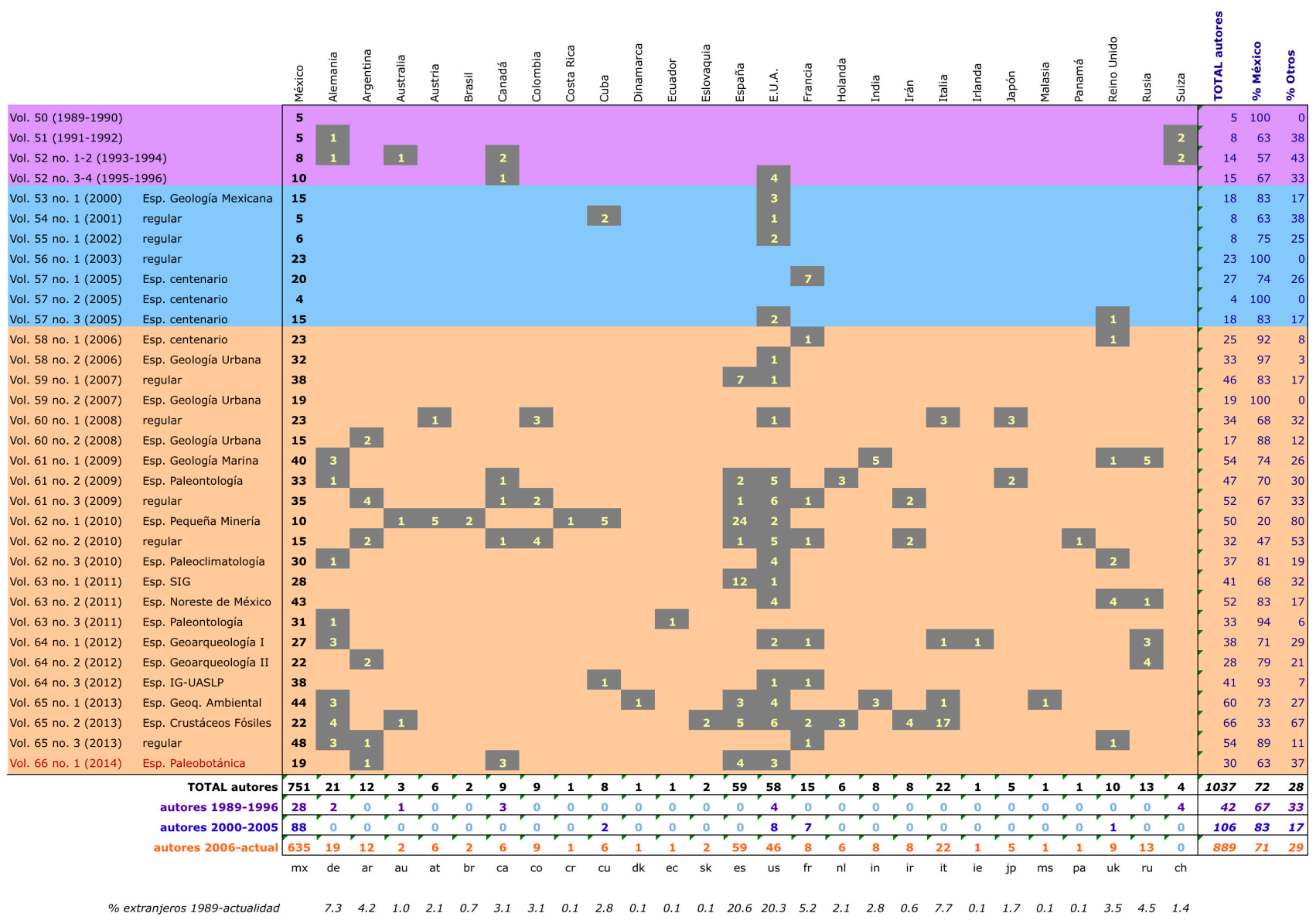

Figura 1. Cuadro de resumen de la procedencia de los autores publicados en el Boletín de la Sociedad Geológica Mexicana desde 1989, por cada número publicado y por la procedencia (país) de la entidad de adscripción de los autores. Los colores denotan el ejercicio de los diversos comités editoriales del Boletín; en naranja, comité editorial actual, durante cuyo ejercicio ha habido, hasta el momento, autores publicados de 26 países diferentes. 
un aumento en la difusión del conocimiento en Ciencias de la Tierra de México y el mundo, contribuyendo así a los estatutos de la Sociedad Geológica Mexicana.

13. Se registra un aumento sustancial en autorías de estudiantes, motivado en gran medida por la aceptación del Boletín en los programas de posgrado como una revista científica rigurosa, y sus contenidos como válidos para obtener una titulación.

14. Se percibe al Boletín como una alternativa interesante y de prestigio para la publicación científica.

15. Se garantiza la continuidad del Boletín como revista científica arbitrada con el mantenimiento y con margen de mejora de su línea editorial.

Todo ello atestigua el establecimiento efectivo del Boletín como una revista científica con arbitraje experto y riguroso, moderna, eficiente, y de carácter generalista dentro del ámbito de las Ciencias de la Tierra. Llegados a este punto, los retos que afronta el Boletín a partir de este momento son de mayor calado, y pudieran llegar a conllevar modificaciones en su línea editorial. Efectivamente, es previsible que la entrada del Boletín al SCI implique un aumento considerable en el número de contribuciones espontáneas que la revista va a estar recibiendo. Ante tal escenario, hay dos posibles consecuencias: (1) aumentar el índice de rechazo endureciendo los arbitrajes — sin que por ello se afecte a manuscritos de suficiente calidad para ameritar su publicación, lo cual constituye un difícil reto-, o (2) aumentar el tiraje de la revista. $\mathrm{O}$ ambas. En cualquiera de estos casos, es preciso redoblar esfuerzos, a fin de que el Boletín no pierda su razón de ser, que no es otra que ofrecer un foro inclusivo (en todos los aspectos posibles) en las Ciencias de la Tierra para la publicación de trabajos científicos relevantes a cualquier escala de observación o análisis, y de contribuir al aumento del conocimiento geológico en México y a su difusión.

Así pues, ¿cuál es el futuro del Boletín en tanto que revista científica arbitrada? Ciertamente, es una revista que debe mantener su foco principal en fomentar la publicación de trabajos relativos a las Ciencias de la Tierra en México,

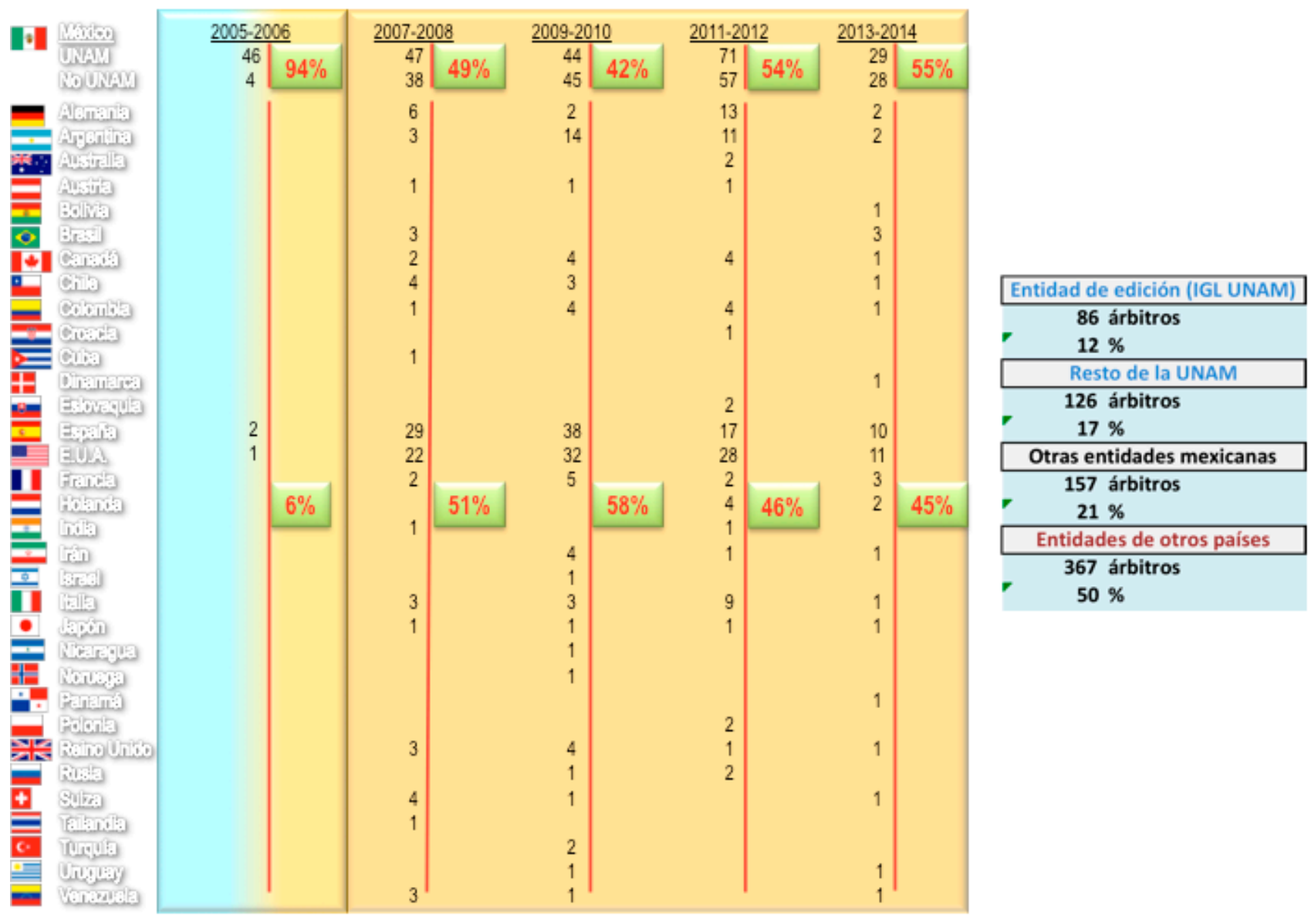

Figura 2. Extracto del padrón de revisores de artículos del Boletín de la Sociedad Geológica Mexicana, a finales de Marzo de 2014 , por bienios y por países. El cambio de color en el bienio 2005-2006 denota el cambio de comité editorial. A la derecha, cuadro de resumen separando los revisores procedentes de la entidad en que se edita la revista (Instituto de Geología de la UNAM), del resto de dependencias de la UNAM, del resto de entidades mexicanas, y de entidades de otros países. 
pues así lo exigen los estatutos de la Sociedad Geológica Mexicana, pero ello no está en contradicción con fomentar la publicación de temas no directamente relacionados con México. La Geología no conoce de fronteras políticas. Por otro lado, el carácter internacional que ya tiene el Boletín en todas las facetas de su edición científica no admite postulados reduccionistas en el ámbito geográfico, y menos aún en el geológico. No se puede concebir el futuro del Boletín sin continuar incidiendo en su carácter generalista e inclusivo. Temáticamente hablando, en el Boletín se tiene especial interés en la publicación de trabajos sobre temas de alcance semi-regional o local, de carácter no necesariamente multimetodológico, y en atención a problemas de Ciencia Aplicada, sin desdeñar trabajos bajo otros enfoques. De este modo, el Boletín lleva ya varios años editando números especiales de artículos de cariz relativamente técnico o de Ciencia Aplicada. Tal es el caso, por ejemplo, de los números especiales de Geología Urbana, de Sistemas de Información Geográfica y Teledetección, o de Geoquímica Ambiental. Por otro lado, también se han editado números especiales de Ciencia Básica, con temas como Geología Regional, Paleoclimatología, Geoarqueología y Paleopedología, y varios números monográficos de Paleontología.

En lo que respecta al idioma de publicación de las contribuciones al Boletín cabe señalar que, desde 2005, un $66 \%$ de los artículos publicados lo han sido en español y un $34 \%$ en inglés. No habrá cambios en la política editorial del Boletín: las lenguas de trabajo seguirán siendo la española y la inglesa. La elección de dicha política no es fútil ni arbitraria, sino que está motivada por (1) el carácter abierto y plural del Boletín, (2) la existencia de temas de trabajo en los que el canal de expresión más apropiado es una u otra lengua, y (3) el hecho de que los índices e instrumentos bibliográficos internacionales requieren de, al menos, un resumen en inglés, y siempre se valora positivamente la presencia de artículos completos en esa lengua. Es decir, la política editorial del Boletín en referencia a las lenguas de trabajo se fundamenta en directrices (internas o externas) de inclusividad, pertinencia y conveniencia. Ello requiere de una explicación adicional: en temas de interés local o semi-regional, el canal de expresión más adecuado suele ser la lengua principal en dicho ámbito geográfico, mientras que dicho canal de expresión es más variable en temas con una orientación más general. Ello, naturalmente, sin coartar en absoluto la libertad de elección de los autores.

El Boletín se ha revelado en los últimos años como un foro altamente adecuado para la publicación de trabajos de estudiantes (principalmente de posgrado) que con frecuencia se encuentran en la etapa inicial de su andadura en la publicación de artículos científicos. Efectivamente, desde 2005 han publicado sus trabajos en el Boletín, por mencionar sólo el caso más relevante, hasta 38 estudiantes del Programa de Posgrado en Ciencias de la Tierra de la Universidad Nacional Autónoma de México - lo cual le confiere ordinalmente a dicha adscripción la cuarta plaza dentro del conjunto de autores publicados - y una cantidad importante de dichos estudiantes han logrado titularse de doctorado mediante estas publicaciones. La elección de la lengua o lenguas de trabajo, por cierto, no parece tener un peso específico importante en el nivel de impacto de las revistas científicas mexicanas que son temáticamente afines al Boletín. Así, los índices de impacto actuales de revistas que publican sus contenidos (1) sólo en inglés, (2) indistintamente en español o inglés, o (3) en ambas lenguas, no son significativamente distintos, al menos en los correspondientes al año 2012 (Figura 3). El idioma de elección no es la única variable de peso que determina qué tan citada puede ser una publicación, aunque es indiscutible que sigue determinando qué tan amplio puede ser el conjunto de lectores potenciales. En ese sentido, lo ideal sería publicar todos los contenidos del Boletín en español y en inglés, algo que no es todavía factible a causa de las limitaciones actuales en cuanto a presupuesto y disponibilidad de personal.

No es posible aventurar cuáles pueden ser los índices de impacto (SCI, SJR, SNIP, etc.) del Boletín en años venideros, pues ello dependerá de qué tan citados sean los trabajos publicados recientemente y en el futuro inmediato, y es un hecho que los valores bibliométricos de las revistas científicas experimentan fluctuaciones considerables de un año a otro. Como ejemplo de ello, considérese la evolución del índice SNIP correspondiente al Boletín: 0.022 en 2009 , 0.405 en 2010, 0.310 en 2011, y 0.621 en 2012 (datos de Scopus). Sin embargo, puede apreciarse en la Figura 3 que los puntajes actuales del Boletín en SJR y SNIP no son, en principio, muy distintos de los del resto de revistas mexicanas de Ciencias de la Tierra que se encuentran desde hace años en el SCI y otros índices internacionales. Ello, a pesar de que la incorporación del Boletín a dichos índices es muy reciente respecto a la de otras revistas mexicanas.

Abundando en lo relativo a la visibilidad del Boletín y de sus contenidos, a pesar de que todavía hay bastante margen de mejora, cabe señalar que actualmente está trabajándose para convertir dichos contenidos a formato HTML y en la extracción de metadatos de los mismos, a fin de facilitar búsquedas en línea. Sin embargo, se dispone en línea de los contenidos íntegros del Boletín desde 1904 en formato PDF. En el momento en que se está elaborando la presente editorial, en nuestra página $\mathrm{web}^{1}$ se encuentran en formato HTML los contenidos completos de los 3 números de 2013 y, parcialmente, del primer número de ese año. Paralelamente, se encuentran en HTML los contenidos del Boletín en su página de $\mathrm{SciELO}^{2}$ desde el ingreso de la revista en sus bases de datos. La conversión a HTML de

\footnotetext{
${ }^{1}$ http://boletinsgm.igeolcu.unam.mx/bsgm/index.php/volumenes-volumes/cuarta-epoca

${ }^{2} \mathrm{http}$ ://www.scielo.org.mx/scielo.php?script=sci_issues\&pid=1405-3322\&lng=es\&nrm=iso
} 

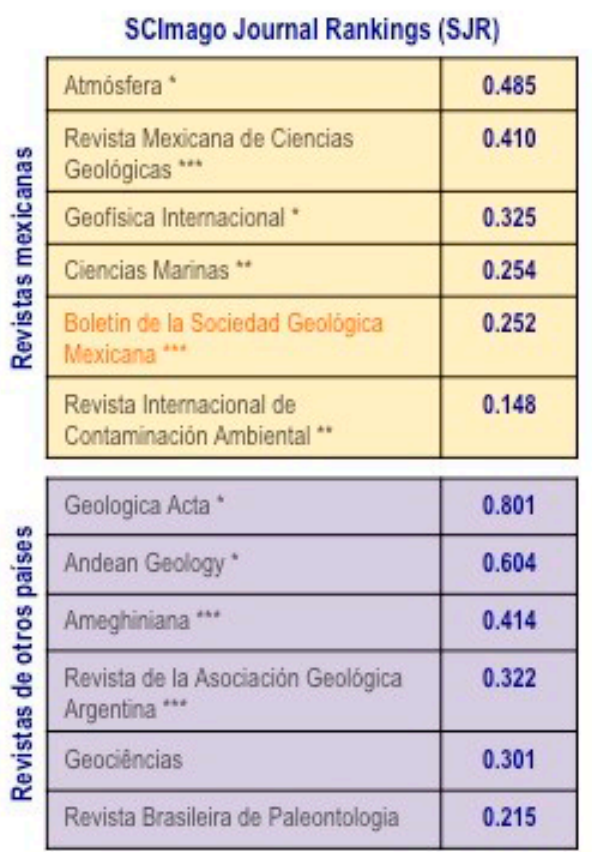

Source Normalized Impact per Paper (SNIP)

\begin{tabular}{|l|c|}
\hline $\begin{array}{l}\text { Revista Mexicana de Ciencias } \\
\text { Geológicas *.. }\end{array}$ & 0.736 \\
\hline Atmósfera * & 0.690 \\
\hline $\begin{array}{l}\text { Boletin de la Sociedad Geológica } \\
\text { Mexicana *. }\end{array}$ & 0.621 \\
\hline Ciencias Marinas ** & 0.502 \\
\hline Geofisica Internacional * & 0.436 \\
\hline $\begin{array}{l}\text { Revista Internacional de } \\
\text { Contaminación Ambiental ** }\end{array}$ & 0.286 \\
\hline
\end{tabular}

\begin{tabular}{|l|c|}
\hline Geologica Acta * & 0.964 \\
\hline Andean Geology * & 0.804 \\
\hline Geociências & 0.704 \\
\hline Ameghiniana *.. & 0.697 \\
\hline $\begin{array}{l}\text { Revista de la Asociación Geológica } \\
\text { Argentina }\end{array}$ & 0.410 \\
\hline Revista Brasileira de Paleontologia & 0.369 \\
\hline
\end{tabular}

Science Citation Index (SCI)

\begin{tabular}{|l|c|}
\hline Atmósfera * & 0.750 \\
\hline Ciencias Marinas ** & 0.609 \\
\hline $\begin{array}{l}\text { Revista Mexicana de Ciencias } \\
\text { Geológicas *.* }\end{array}$ & 0.505 \\
\hline $\begin{array}{l}\text { Revista Internacional de } \\
\text { Contaminaciön Ambiental ** }\end{array}$ & 0.257 \\
\hline Geofisica Internacional * & 0.220 \\
\hline $\begin{array}{l}\text { Boletin de la Sociedad Geológica } \\
\text { Mexicana *.. }\end{array}$ & - \\
\hline
\end{tabular}

\begin{tabular}{|l|c|}
\hline Andean Geology * & 1.375 \\
\hline Geologica Acta * & 1.262 \\
\hline Ameghiniana *.. & 0.866 \\
\hline Geociências & - \\
\hline Revista Brasileira de Paleontologia & - \\
\hline $\begin{array}{l}\text { Revista de la Asociación Geológica } \\
\text { Argentina *.. }\end{array}$ & - \\
\hline
\end{tabular}

"Publica articulos sólo en inglés. "* Publica articulos en inglés y español. ** Publica articulos en inglés o en español.

Figura 3. Comparación entre diversos índices de impacto internacionales en las revistas mexicanas del área de Ciencias de la Tierra indizadas, en comparación con algunas revistas de Latinoamérica o España que son afines temáticamente a los contenidos del Boletín de la Sociedad Geológica Mexicana. Datos procedentes de SCImago (SJR), Scopus (SNIP) y, Journal Citation Reports (SCI) correspondientes al año 2012.

los contenidos del Boletín continuará progresando hacia números pasados, mientras que todos los contenidos de nueva factura aparecerán simultáneamente en formato PDF y HTML. Al margen de la futura exigencia por parte del CONACYT de que las revistas incluidas en su índice dispongan de sus contenidos en formato HTML, es preciso recordar que dicho formato es particularmente útil para facilitar la visibilidad de las revistas en la red mundial.

En un número futuro, estaremos realizando un reporte más pormenorizado de los avances en la edición del Boletín, al estilo del que se presentó en el vol. 61 no. 2 de 2009, en forma de editorial. Por lo pronto, celebremos la feliz confluencia de la inclusión de esta revista en el SCI con su $110^{\circ}$ aniversario.
Antoni Camprubí

Editor en jefe

Ciudad de México, 30 de Marzo de 2014 